\title{
Correction to: In-depth characterization of the tumor microenvironment in central nervous system lymphoma reveals implications for immune-checkpoint therapy
}

\author{
Lukas Marcelis $^{1}$ - Asier Antoranz ${ }^{1}$ - Anne-Marie Delsupehe ${ }^{2}$. Pauline Biesemans ${ }^{1}$. Julio Finalet Ferreiro ${ }^{3}$. \\ Koen Debackere ${ }^{4}$ Peter Vandenberghe ${ }^{5}$. Gregor Verhoef ${ }^{4,5} \cdot$ Paul Clement $^{6} \cdot$ Steven De Vleeschouwer ${ }^{7,8}$. \\ Olivier Gheysens ${ }^{9} \cdot$ Giorgio Cattoretti $^{10,11}$. Francesca Maria Bosisio ${ }^{1,2} \cdot$ Xavier Sagaert $^{1,2}$. Daan Dierickx ${ }^{4,5}$. \\ Thomas Tousseyn ${ }^{1,2}$
}

Published online: 17 February 2021

(c) Springer-Verlag GmbH Germany, part of Springer Nature 2021

\section{Correction to: \\ Cancer Immunology and Immunotherapy (2020) 69:1751-1766 \\ https://doi.org/10.1007/s00262-020-02575-y}

The original version of this article unfortunately contained a mistake. Paul Clement and Steven De Vleeschouwer were not listed among the authors. The correct information is given below.

Lukas Marcelis ${ }^{1}$, Asier Antoranz ${ }^{1}$, Anne-Marie Delsupehe $^{2}$, Pauline Biesemans ${ }^{1}$, Julio Finalet Ferreiro ${ }^{3}$, Koen Debackere ${ }^{4}$, Peter Vandenberghe ${ }^{5}$, Gregor Verhoef ${ }^{4,5}$, Paul Clement ${ }^{6}$, Steven De Vleeschouwer ${ }^{7,8}$, Olivier Gheysens $^{9}$, Giorgio Cattoretti ${ }^{10,11}$, Francesca Maria Bosisio $^{1,2}$, Xavier Sagaert ${ }^{1,2}$, Daan Dierickx ${ }^{4,5}$, Thomas Tousseyn ${ }^{1,2}$

1. KU Leuven, Department of Imaging and Pathology, Translational Cell and Tissue Research Lab, Leuven, Belgium

2. UZ Leuven, University Hospitals, Department of Pathology, Leuven, Belgium

The original article can be found online at https://doi.org/10.1007/ s00262-020-02575-y.

Thomas Tousseyn

thomas.tousseyn@uzleuven.be

1 Translational Cell and Tissue Research Lab, Department of Imaging and Pathology, KU Leuven, Leuven, Belgium

2 Department of Pathology, University Hospitals, UZ Leuven, Leuven, Belgium

3 Center of Human Genetics, KU Leuven, Leuven, Belgium

4 Laboratory of Experimental Hematology, Department of Oncology, KU Leuven, Leuven, Belgium

5 Department of Hematology, University Hospitals, UZ Leuven, Leuven, Belgium
3. KU Leuven, Center of Human Genetics, Leuven, Belgium

4. KU Leuven, Department of Oncology, Laboratory of Experimental Hematology

5. UZ Leuven, University Hospitals, Department of Hematology, Leuven, Belgium

6. KU Leuven, Leuven Cancer institute, Department of Oncology, Leuven, Belgium

7. UZ Leuven, University Hospitals,Department of Neurosurgery, Leuven, Belgium

8. KU Leuven, Leuven Brain institute, Leuven, Belgium

9. UZ Leuven, University Hospitals, Department of Nuclear Medicine, Leuven, Belgium

10. Università degli Studi di Milano-Bicocca, Department of Medicine and Surgery, Anatomic Pathology, Milano, Italy

11. ASST-Monza, Ospedale San Gerardo, Department of Pathology, Italy

Publisher's Note Springer Nature remains neutral with regard to jurisdictional claims in published maps and institutional affiliations.

6 Department of Oncology, Leuven Cancer Institute, KU Leuven, Leuven, Belgium

7 Department of Neurosurgery, University Hospitals, UZ Leuven, Leuven, Belgium

8 Leuven Brain Institute, KU Leuven, Leuven, Belgium

9 Department of Nuclear Medicine, University Hospitals, UZ Leuven, Leuven, Belgium

10 Department of Medicine and Surgery, Anatomic Pathology, Università Degli Studi Di Milano-Bicocca, Milano, Italy

11 Department of Pathology, Ospedale San Gerardo, ASST-Monza, Monza, Italy 